Callao, V. \& Montoya, E. (1960). J. gen. Microbiol. 22, 657-661

\title{
The Use of Dyes to Distinguish Between Species of the Genus Azotobacter
}

\author{
By V. CALLAO and E. MONTOYA \\ Estacion Experimental del Zaidin, Granada, Spain
}

SUMMARY: The effects of several dyes on the growth on solid culture media of several strains of $A$ zotobacter chroococcum, $A$. vinelandii, $A$. agile and $A$. beijerinckii were studied. Some of the dyes inhibited the growth of one or more of the Azotobacter spp. tested. From our results, we conclude that $A$. chroococcum, $A$. vinelandii, $\boldsymbol{A}$. agile and $\boldsymbol{A}$. beijerinckii are different species, well characterized by their behaviour to the dyes. We propose a scheme, based on the action of $1 / 25,000(w / v)$ Pyronine and 1/50,000 (w/v) Diamond fuchsin which differentiates the four Azotobacter species quickly and easily.

The family Azotobacteriaceae includes a group of organisms which are Gramnegative, non-sporeforming aerobes able to fix atmospheric nitrogen without a symbiotic life. They have large oval cells, usually in pairs, often forming quantities of gum. These characters permit a rapid classification of organism in this family, but the differentiation of species and genera within the group is still a problem.

At the present time, there are two alternative schemes for the Azotobacteriaceae family. One, given by Hofer in Bergey's Manual (1957), admits one genus, Azotobacter, which includes three species: $A$. chroococcum, A. agile and $A$. indicus. The other scheme, given by Jensen (1954) and Pochon (Pochon \& Barjac, 1958) has two genera: Azotobacter and Beijerinckia. Jensen's genus Azotobacter has four species: $A$. chroococcum, $A$. vinelandii, $A$. agile and $\boldsymbol{A}$. beijerinckii; Pochon only accepts two species, $A$. chroococcum and $A$. agile, and considers that $A$. vinelandii and $A$. beijerinckii are varieties of the preceding species. Both Jensen and Pochon include in the genus Beijerinckia the species Azotobacter indicus, Starkey and De, and other varieties.

We found that the Azotobacter are very sensitive to the bacteriostatic action of certain dyes, and this paper is an account of experiments applying to the genus Azotobacter the same techniques that are used for the differentiation of the genus Brucella (Huddleson, 1934).

\section{METHODS}

Organisms. Typical strains of Azotobacter chroococcum, strain 0-1, A. vinelandii, strain 0-2, $A$. agile, strain 0-3, and $A$. beijerinckii, strain 0-4, obtained from the Pasteur Institute, Paris, were used.

The results obtained were checked with five other strains of Azotobacter chroococcum (nos. 17, 55, 103, 331 and 567), three strains of $A$. vinelandii (nos.615,621, and 668), two strains of $A$. agile (nos. 44 and 573) and eight strains of $A$. beijerinckii (nos. 11, 12, 332, 546, 566, 586, 592 and 597 ), isolated in our 
laboratory in the last two years from the soils and the waters of Granada province and classified according to the techniques of Jensen (1954) and Jensen \& Petersen (1955).

Table 1 shows the main characters of the Azotobacter strains used.

Table 1. Pigmentation, mobility, formation of cysts, lower $\mathrm{pH}-$ limit for growth and ability to utilize various sources of carbon, of the Azotobacter used

\begin{tabular}{|c|c|c|c|c|c|c|c|c|c|c|c|c|c|c|c|c|c|}
\hline Species & 点 & 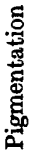 & 胥 & 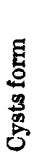 & 范 & 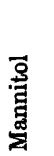 & 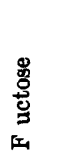 & 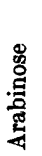 & 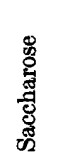 & 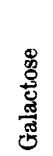 & 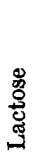 & 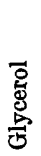 & 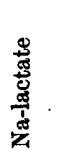 & 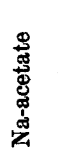 & 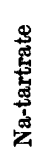 & 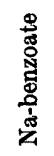 & 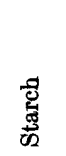 \\
\hline A. chroococcum & $\begin{array}{r}0-1 \\
17 \\
55 \\
103 \\
331 \\
567\end{array}$ & $\begin{array}{l}\mathbf{b} \\
\mathbf{b} \\
\mathbf{b} \\
\mathbf{b} \\
\mathbf{b} \\
\mathbf{b}\end{array}$ & $\begin{array}{l}\mathbf{m} \\
\mathbf{m} \\
\mathbf{m} \\
\mathbf{m} \\
\mathbf{m} \\
\mathbf{m}\end{array}$ & $\begin{array}{l}\mathrm{c} \\
\mathrm{cc} \\
\mathrm{c} \\
\mathrm{c} \\
\mathrm{c} \\
\mathrm{cc}\end{array}$ & $\begin{array}{l}5 \cdot 7 \\
5 \cdot 6 \\
5 \cdot 2 \\
5 \cdot 8 \\
5 \cdot 7 \\
5 \cdot 6\end{array}$ & $\begin{array}{l}++ \\
++ \\
+ \\
+ \\
+ \\
+\end{array}$ & $\begin{array}{l}+ \\
+ \\
+ \\
+ \\
+ \\
++\end{array}$ & $\begin{array}{l}+ \\
+ \\
- \\
- \\
-\end{array}$ & $\begin{array}{c}++ \\
++ \\
+ \\
+ \\
+ \\
+\end{array}$ & $\begin{array}{c}++ \\
+ \\
+ \\
+ \\
++ \\
++\end{array}$ & $\begin{array}{l}- \\
- \\
- \\
- \\
-\end{array}$ & $\begin{array}{l}- \\
- \\
- \\
+ \\
+ \\
+\end{array}$ & $\begin{array}{c}++ \\
++ \\
+ \\
++ \\
++ \\
++\end{array}$ & $\begin{array}{l}++ \\
++ \\
++ \\
++ \\
++ \\
++\end{array}$ & $\begin{array}{l}- \\
+ \\
+ \\
+ \\
+\end{array}$ & $\begin{array}{l}+ \\
+ \\
+ \\
+ \\
++ \\
+\end{array}$ & $\begin{array}{l}+t \\
+t \\
+t \\
++ \\
+t \\
+t\end{array}$ \\
\hline A. vinelandii & $\begin{array}{l}0-2 \\
615 \\
621 \\
668\end{array}$ & $\begin{array}{l}\text { yg } \\
\text { yg } \\
\text { yg } \\
\text { yg }\end{array}$ & $\begin{array}{l}\mathrm{m} \\
\mathrm{mm} \\
\mathrm{m} \\
\mathrm{mm}\end{array}$ & $\begin{array}{l}\mathrm{cc} \\
\mathrm{cc} \\
\mathrm{cc} \\
\mathrm{cc}\end{array}$ & $\dot{.}$ & $\begin{array}{l}++ \\
++ \\
++ \\
++\end{array}$ & $\begin{array}{c}+ \\
++ \\
++ \\
+\end{array}$ & $\frac{-}{+}$ & $\begin{array}{c}++ \\
+ \\
+ \\
+\end{array}$ & $\begin{array}{c}++ \\
+ \\
++ \\
++\end{array}$ & $\begin{array}{l}+ \\
+ \\
+ \\
+\end{array}$ & $\begin{array}{l}+ \\
+ \\
+ \\
+\end{array}$ & $\begin{array}{l}- \\
- \\
-\end{array}$ & $\begin{array}{c}+ \\
+ \\
++ \\
+\end{array}$ & $\begin{array}{l}- \\
+ \\
-\end{array}$ & $\begin{array}{c}+ \\
++ \\
++ \\
+\end{array}$ & $\begin{array}{l}- \\
- \\
-\end{array}$ \\
\hline A. agile & $\begin{array}{r}0-3 \\
44 \\
573\end{array}$ & $\begin{array}{l}\text { yg } \\
\text { yg } \\
\text { yg }\end{array}$ & $\begin{array}{l}\mathrm{mm} \\
\mathrm{mm} \\
\mathrm{mm}\end{array}$ & $\begin{array}{l}\text { nc } \\
\text { nc } \\
\text { nc }\end{array}$ & $\begin{array}{l}. \\
. \\
.\end{array}$ & $\overline{-}$ & $\begin{array}{c}+ \\
+ \\
+t\end{array}$ & $\begin{array}{l}- \\
-\end{array}$ & $\begin{array}{c}++ \\
+ \\
++\end{array}$ & \pm & $\begin{array}{l}- \\
-\end{array}$ & $\begin{array}{l}+ \\
+ \\
+\end{array}$ & $\begin{array}{l}- \\
+\end{array}$ & $\begin{array}{l}+ \\
+ \\
+\end{array}$ & $\begin{array}{l}- \\
-\end{array}$ & $\begin{array}{l}+ \\
+ \\
+\end{array}$ & $\frac{-}{-}$ \\
\hline A. beijerinckii & $\begin{array}{r}0-4 \\
11 \\
12 \\
332 \\
546 \\
566 \\
586 \\
592 \\
597\end{array}$ & $\begin{array}{l}n p \\
n p \\
n p \\
n p \\
n p \\
n p \\
y \\
y \\
n p\end{array}$ & $\begin{array}{l}\mathrm{nm} \\
\mathrm{nm} \\
\mathrm{nm} \\
\mathrm{nm} \\
\mathrm{nm} \\
\mathrm{nm} \\
\mathrm{nm} \\
\mathrm{nm} \\
\mathrm{nm}\end{array}$ & $\begin{array}{l}\text { c } \\
\text { nc } \\
? \\
\text { c } \\
? \\
\text { nc } \\
\text { nc } \\
? \\
\text { c }\end{array}$ & $\begin{array}{l}\mathbf{5} \cdot 0 \\
\mathbf{5} \cdot \mathbf{2} \\
\mathbf{5} \cdot 1 \\
\mathbf{5} \cdot \mathbf{3} \\
\mathbf{5 \cdot 2} \\
\mathbf{5} \cdot 2 \\
\mathbf{5 \cdot 2} \\
\mathbf{5} \cdot 2 \\
\mathbf{5 \cdot 1}\end{array}$ & $\begin{array}{c}++ \\
+ \\
++ \\
+ \\
++ \\
++ \\
++ \\
++ \\
++\end{array}$ & $\begin{array}{c}++ \\
++ \\
++ \\
++ \\
++ \\
+ \\
+ \\
+ \\
++\end{array}$ & $\begin{array}{l}+ \\
+ \\
+ \\
+ \\
+ \\
+ \\
+ \\
+ \\
+\end{array}$ & $\begin{array}{c}++ \\
+ \\
+ \\
+ \\
+ \\
+ \\
++ \\
++ \\
++\end{array}$ & $\begin{array}{l}+ \\
- \\
- \\
- \\
- \\
- \\
-\end{array}$ & $\begin{array}{l}- \\
- \\
- \\
- \\
- \\
- \\
-\end{array}$ & $\begin{array}{l}- \\
- \\
\bar{t} \\
+ \\
+ \\
+ \\
-\end{array}$ & $\begin{array}{c}- \\
- \\
\overrightarrow{+} \\
++ \\
++ \\
+ \\
++ \\
+\end{array}$ & $\begin{array}{c}++ \\
++ \\
+ \\
+ \\
++ \\
+ \\
+ \\
++ \\
++\end{array}$ & $\begin{array}{l}- \\
- \\
- \\
+ \\
- \\
- \\
-\end{array}$ & $\begin{array}{c}++ \\
+ \\
++ \\
++ \\
++ \\
++ \\
++ \\
++ \\
+t\end{array}$ & $\begin{array}{l}\overline{+} \\
- \\
- \\
\bar{t} \\
+ \\
-\end{array}$ \\
\hline
\end{tabular}

Glucose, maltose and ethanol ++ for all the strains tested. Xylose, mannose and Na-citrate - for all the strains tested. Pigmentation: b, brown to black; yg, yellow-greenish; y, yellowish; np, colourless.

Mobility: $\mathrm{mm}$, strong; $\mathrm{m}$, moderate; $\mathrm{nm}$, negative.

Formation of cysts: cc, abundant; c, slight; ?, questionable; nc, negative.

Growth: ++ , strong; + , moderate; - , questionable or none.

Media. We used the ' 77 ' solid medium of Allen (1951). To improve the growth of Azotobacter agile, mannitol was replaced by a mixture of $0.5 \%$ $(\mathrm{w} / \mathrm{v})$ glucose and $0.5 \%(\mathrm{w} / \mathrm{v})$ maltose.

Dyes. The following dyes were used: Bismark brown (Geigy), Alcaline blue B (Grübler), Cotton blue (Geigy), Methylene blue (Geigy), Carmine nacarat (Merck), Acid fuchsin (B.D.H.), Diamond fuchsin (Merck), Brilliant green (Sandoz), Malachite green (Merck), Methyl green (Merck), Pyronine (Geigy), Congo red (Merck), Neutral red (Geigy), Phenol red (Grübler), Safranine T (Merck), Sudan III (Geigy), Thionine (Geigy), Violamine 4B (Chroma), Crystal violet (Merck) and Methyl violet (Geigy).

Diamond fuchsin, Sudan III, Crystal violet and Methyl violet were prepared in ethanolic solution, at a concentration of $0 \cdot 2 \%(\mathrm{w} / \mathrm{v})$, and the remaining dyes in aqueous solution at the same concentration. Each dye was distributed in $5 \mathrm{ml}$. vials, and sterilized at $100^{\circ}$ for $1 \mathrm{hr}$.

Proceeding. Measured volumes of each dye were added to flasks containing $100 \mathrm{ml}$. of 77 -agar to give final concentrations of $1 / 25,000 ; 1 / 50,000 ; 1 / 100,000$ and $1 / 200,000(w / v)$. The flasks were agitated and the contents poured into 
four sterile Petri dishes of $100 \mathrm{~mm}$. diameter. Plates were inoculated by dropping into the surface a $48 \mathrm{hr}$. culture of the required Azotobacter. The plates were then incubated at $30^{\circ}$, and growth was recorded at 48,72 and $96 \mathrm{hr}$.

Table 2. Action of some dyes, at different concentrations, on the growth of the Azotobacter

\begin{tabular}{|c|c|c|c|c|c|c|c|c|c|c|c|c|c|}
\hline \multirow[b]{2}{*}{ Dye } & \multirow{2}{*}{$\begin{array}{c}\text { Concentra- } \\
\text { tion } \\
(\mathbf{w} / \mathbf{v})\end{array}$} & \multicolumn{3}{|c|}{$\begin{array}{c}\text { A. chroococcum } \\
0-1\end{array}$} & \multicolumn{3}{|c|}{$\begin{array}{c}\text { A. vinelandii } \\
0-2\end{array}$} & \multicolumn{3}{|c|}{ A. agile } & \multicolumn{3}{|c|}{ A. beijerinckii } \\
\hline & & $48 \mathrm{hr}$. & $72 \mathrm{hr}$ & $96 \mathrm{hr}$. & $48 \mathrm{hr}$ & $72 \mathrm{hr}$. & $96 \mathrm{hr}$. & $48 \mathrm{hr}$. & $72 \mathrm{hr}$. & $96 \mathrm{hr}$. & $48 \mathrm{hr}$. & $72 \mathrm{br}$. & $96 \mathrm{hr}$. \\
\hline $\begin{array}{l}\text { Bisniark- } \\
\text { brown }\end{array}$ & $\begin{array}{l}1 / 25,000 \\
1 / 50,000 \\
1 / 100,000\end{array}$ & $\begin{array}{l}- \\
+ \\
+\end{array}$ & $\begin{array}{l}\overline{+} \\
+\end{array}$ & $\begin{array}{l}+ \\
+ \\
+\end{array}$ & $\begin{array}{l}+ \\
+ \\
+\end{array}$ & $\begin{array}{l}+ \\
+ \\
+\end{array}$ & $\begin{array}{l}+ \\
+ \\
+\end{array}$ & $\begin{array}{l}- \\
+ \\
+\end{array}$ & $\begin{array}{l}+ \\
+ \\
+\end{array}$ & $\begin{array}{l}+ \\
+ \\
+\end{array}$ & $\frac{-}{+}$ & $\frac{-}{+}$ & $\begin{array}{l}- \\
+ \\
+\end{array}$ \\
\hline $\begin{array}{l}\text { Diamond } \\
\text { fuchsin }\end{array}$ & $\begin{array}{l}1 / 25,000 \\
1 / 50,000 \\
1 / 100,000 \\
1 / 200,000\end{array}$ & $\begin{array}{l}- \\
\bar{t}\end{array}$ & 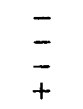 & $\begin{array}{l}- \\
\bar{t}\end{array}$ & $\begin{array}{l}- \\
+ \\
+ \\
+\end{array}$ & $\begin{array}{l}+ \\
+ \\
+ \\
+\end{array}$ & $\begin{array}{l}+ \\
+ \\
+ \\
+\end{array}$ & $\begin{array}{l}+ \\
+ \\
+ \\
+\end{array}$ & $\begin{array}{l}+ \\
+ \\
+ \\
+\end{array}$ & $\begin{array}{l}+ \\
+ \\
+ \\
+\end{array}$ & $\begin{array}{l}- \\
\overline{-} \\
+\end{array}$ & $\begin{array}{l}- \\
\bar{t} \\
+\end{array}$ & $\begin{array}{l}- \\
- \\
+\end{array}$ \\
\hline $\begin{array}{l}\text { Brilliant } \\
\text { green }\end{array}$ & $\begin{array}{l}1 / 25,000 \\
1 / 50,000 \\
1 / 100,000 \\
1 / 200,000\end{array}$ & $\begin{array}{l}- \\
\bar{t}\end{array}$ & $\begin{array}{l}- \\
\bar{t}\end{array}$ & $\begin{array}{l}- \\
\bar{t}\end{array}$ & $\begin{array}{l}- \\
\bar{t}\end{array}$ & $\frac{-}{-}$ & $\overline{-}$ & $\begin{array}{l}+ \\
+ \\
+ \\
+\end{array}$ & $\begin{array}{l}+ \\
+ \\
+ \\
+\end{array}$ & $\begin{array}{l}+ \\
+ \\
+ \\
+\end{array}$ & $\begin{array}{l}- \\
\bar{t}\end{array}$ & $\begin{array}{l}- \\
\bar{t}\end{array}$ & $\begin{array}{l}- \\
+ \\
+\end{array}$ \\
\hline $\begin{array}{l}\text { Malachite } \\
\text { green }\end{array}$ & $\begin{array}{l}1 / 25,000 \\
1 / 50,000 \\
1 / 100,000 \\
1 / 200,000\end{array}$ & $\begin{array}{l}- \\
\overrightarrow{+}\end{array}$ & $\overline{-}$ & $\begin{array}{l}- \\
\bar{t}\end{array}$ & $\begin{array}{l}+ \\
+ \\
+ \\
+\end{array}$ & $\begin{array}{l}+ \\
+ \\
+ \\
+\end{array}$ & $\begin{array}{l}+ \\
+ \\
+ \\
+\end{array}$ & $\begin{array}{l}+ \\
+ \\
+ \\
+\end{array}$ & $\begin{array}{l}+ \\
+ \\
+ \\
+\end{array}$ & $\begin{array}{l}+ \\
+ \\
+ \\
+\end{array}$ & $\begin{array}{l}- \\
\bar{t} \\
+\end{array}$ & $\begin{array}{l}\overline{-} \\
\bar{t} \\
+\end{array}$ & $\begin{array}{l}- \\
+ \\
+ \\
+\end{array}$ \\
\hline Pyronine & $\begin{array}{l}1 / 25,000 \\
1 / 50,000 \\
1 / 100,000 \\
1 / 200,000\end{array}$ & $\begin{array}{l}+ \\
+ \\
+ \\
+\end{array}$ & $\begin{array}{l}+ \\
+ \\
+ \\
+\end{array}$ & $\begin{array}{l}+ \\
+ \\
+ \\
+\end{array}$ & $\begin{array}{l}+ \\
+ \\
+ \\
+\end{array}$ & $\begin{array}{l}+ \\
+ \\
+ \\
+\end{array}$ & $\begin{array}{l}+ \\
+ \\
+ \\
+\end{array}$ & $\begin{array}{l}- \\
\bar{t}\end{array}$ & $\begin{array}{l}\overline{-} \\
\bar{t}\end{array}$ & $\begin{array}{l}- \\
\bar{t}\end{array}$ & $\begin{array}{l}- \\
\dot{t} \\
+\end{array}$ & $\begin{array}{l}\bar{t} \\
+ \\
+\end{array}$ & $\begin{array}{l}- \\
+ \\
+ \\
+\end{array}$ \\
\hline Safranine & $\begin{array}{l}1 / 25,000 \\
1 / 50,000 \\
1 / 100,000 \\
1 / 200,000\end{array}$ & $\begin{array}{l}- \\
\bar{t} \\
+\end{array}$ & $\begin{array}{l}- \\
+ \\
+ \\
+\end{array}$ & $\begin{array}{l}- \\
+ \\
+ \\
+\end{array}$ & $\begin{array}{l}+ \\
+ \\
+ \\
+\end{array}$ & $\begin{array}{l}+ \\
+ \\
+ \\
+\end{array}$ & $\begin{array}{l}+ \\
+ \\
+ \\
+\end{array}$ & $\begin{array}{l}+ \\
+ \\
+ \\
+\end{array}$ & $\begin{array}{l}+ \\
+ \\
+ \\
+\end{array}$ & $\begin{array}{l}+ \\
+ \\
+ \\
+\end{array}$ & $\begin{array}{l}- \\
\overline{-} \\
-\end{array}$ & $\overline{-}$ & $\begin{array}{l}- \\
- \\
-\end{array}$ \\
\hline Sudan III & $\begin{array}{l}1 / 25,000 \\
1 / 50,000\end{array}$ & $\begin{array}{l}+ \\
+\end{array}$ & $\begin{array}{l}+ \\
+\end{array}$ & $\stackrel{+}{+}$ & + & + & + & + & + & $\begin{array}{l}+ \\
+\end{array}$ & $\overline{+}$ & $\overline{+}$ & $\bar{t}$ \\
\hline Thionine & $\begin{array}{l}1 / 25,000 \\
1 / 50,000 \\
1 / 100,000\end{array}$ & $\begin{array}{l}\bar{t} \\
+\end{array}$ & $\begin{array}{l}- \\
+\end{array}$ & $\begin{array}{l}+ \\
+ \\
+\end{array}$ & $\begin{array}{l}+ \\
+ \\
+\end{array}$ & $\begin{array}{l}+ \\
+ \\
+\end{array}$ & $\begin{array}{l}+ \\
+ \\
+\end{array}$ & $\begin{array}{l}+ \\
+ \\
+\end{array}$ & $\begin{array}{l}+ \\
+ \\
+\end{array}$ & $\begin{array}{l}+ \\
+ \\
+\end{array}$ & $\bar{t}$ & $\begin{array}{l}- \\
+\end{array}$ & $\begin{array}{l}- \\
+ \\
+\end{array}$ \\
\hline
\end{tabular}

\section{RESULTS}

\section{Action of the dyes on typical strains}

Alcaline blue, Cotton blue, Methylene blue, Carmine nacarat, Acid fuchsin, Methyl green, Congo red, Neutral red, Phenol red and Violamine did not have any effect, at the concentrations used, on the normal growth of any of the Azotobacter strains.

Crystal violet and Methyl violet were completely inhibitory even at a concentration of 1/200,000. Azotobacter chroococcum strain 0-1 and A. vinelandii strain 0-2 occasionally grew on the 77 -agar $+1 / 200,000$ Crystal violet but in this case the growth was slight and did not appear before $96 \mathrm{hr}$.

Bismark brown, Diamond fuchsin, Brilliant green, Malachite green, Pyronine, Safranine, Sudan III and Thionine have a different action on each Azotobacter. Table 2 shows the behaviour of the Azotobacter strains in the presence of these dyes at different concentrations.

From the dyes used (shown in Table 2) we selected the following as being the most useful for the differentiation of Azotobacter. 
1/50,000 Diamond fuchsin and 1/25,000 Malachite green which differentiate Azotobacter chroococcum and $A$. beijerinckii from $A$. vinelandii and A. agile.

$1 / 50,000$ Brilliant green which only permits the growth of $A$. agile.

1/25,000 Pyronine which differentiates $A$. chroococcum and $A$. vinelandii from $A$. agile and $A$. beijerinckii, and

1/100,000 Safranine which differentiates $A$. beijerinckii from the other three.

These dyes were then used at these concentrations on our local strains to check their action on different strains of the same species (Table 3).

Table 3. Effect of Diamond fuchsin 1/50,000, Brilliant green 1/50,000, Malachite green 1/25,000, Pyronine 1/25,000, and Safranine 1/100,000 on the growth of the typical local strains of Azotobacter

\begin{tabular}{|c|c|c|c|c|c|c|}
\hline Species & Strain & $\begin{array}{c}\text { Diamond } \\
\text { fuchsin } \\
1 / 50,000\end{array}$ & $\begin{array}{c}\text { Brilliant } \\
\text { green } \\
1 / 50,000\end{array}$ & $\begin{array}{c}\text { Malachite } \\
\text { green } \\
\mathbf{1 / 2 5 , 0 0 0}\end{array}$ & $\begin{array}{r}\text { Pyronine } \\
\mathbf{1} / \mathbf{2 5 , 0 0 0}\end{array}$ & $\begin{array}{l}\text { Safranine } \\
1 / 100,000\end{array}$ \\
\hline A. chroococcum & $\begin{array}{r}17 \\
55 \\
103 \\
331 \\
567\end{array}$ & $\begin{array}{l}- \\
\bar{z} \\
\overline{-}\end{array}$ & $\begin{array}{l}\overline{-} \\
\overline{-} \\
\overline{-}\end{array}$ & $\begin{array}{l}- \\
\overline{-} \\
\overline{-}\end{array}$ & $\begin{array}{l}+ \\
+ \\
+ \\
+ \\
+\end{array}$ & $\begin{array}{l}+ \\
+ \\
+ \\
+ \\
+\end{array}$ \\
\hline A. vinelandii & $\begin{array}{l}615 \\
621 \\
668\end{array}$ & $\begin{array}{l}+ \\
+ \\
+\end{array}$ & $\begin{array}{l}- \\
-\end{array}$ & $\begin{array}{l}+ \\
+ \\
+\end{array}$ & $\begin{array}{l}+ \\
+ \\
+\end{array}$ & $\begin{array}{l}+ \\
+ \\
+\end{array}$ \\
\hline A. agile & $\begin{array}{r}44 \\
573\end{array}$ & + & $\begin{array}{l}+ \\
+\end{array}$ & $\begin{array}{l}+ \\
+\end{array}$ & - & $\begin{array}{l}+ \\
+\end{array}$ \\
\hline A. beijerinckii & $\begin{array}{r}11 \\
12 \\
\mathbf{3 3 2} \\
546 \\
566 \\
586 \\
592 \\
597\end{array}$ & $\begin{array}{l}\bar{z} \\
\bar{z} \\
z \\
\bar{z} \\
-\end{array}$ & $\begin{array}{l}- \\
- \\
- \\
- \\
- \\
- \\
-\end{array}$ & $\begin{array}{l}- \\
- \\
- \\
- \\
- \\
- \\
-\end{array}$ & $\begin{array}{l}- \\
- \\
- \\
- \\
- \\
- \\
-\end{array}$ & $\begin{array}{l}+ \\
+ \\
- \\
- \\
+ \\
+ \\
- \\
-\end{array}$ \\
\hline
\end{tabular}

\section{DISCUSSION}

Bismark brown, Diamond fuchsin, Brilliant green, Malachite green, Pyronine, Safranine, Sudan III and Thionine, at suitable concentrations, permit the growth of some cultures of Azotobacter but inhibit others. Diamond fuchsin 1/50,000, Brilliant green 1/50,000, Malachite green 1/25,000 and Pyronine $1 / 25,000$ were the most useful.

The reactions of the different Azotobacter species to these dyes has been shown to be sufficiently constant to be useful for the differentiation of species within the genus. In fact, using these four dyes, it was possible to divide Azotobacter into four types as shown below. This, with their other characters, we consider to be adequate evidence that Azotobacter chroococcum, A. vinelandii, $\boldsymbol{A}$. agile, and $\boldsymbol{A}$. beijerinckii are distinct species.

We propose the following scheme, based on the utilization of two dyes, for the differentiation of the Azotobacter genus. 
(1) Growth on solid culture media containing 1/25,000 Pyronine.

(a) No growth on solid culture media with 1/50,000 Diamond fuchsin A. chroococcum

(b) Growth on solid culture media with 1/50,000 Diamond fuchsin

$$
\text { A. vinelandii }
$$

(2) No growth on solid culture media with 1/25,000 Pyronine.

(a) Growth on solid culture media with 1/50,000 Diamond fuchsin.

(b) No growth on solid culture media with $1 / 50,000$ Diamond fuchsin

$$
\text { A. agile }
$$

A. beijerinckii

Malachite green $(1 / 25,000)$ and Brilliant green $(1 / 50,000)$ could be used to confirm these differences.

\section{REFERENCES}

Aluen, O. N. (1951). Experiments in Soil Bacteriology. Burgess Publishing Co., Minnesota., U.S.A.

Hofer, A. W. (1957). In Bergey's Manual of Determinative Bacteriology, 7th ed. Eds. R. S. Breed, E. D. G. Murray \& N. R. Smith, p. 283. London: Baillière, Tindall and Cox, Ltd.

Huddleson, I. F. (1934). Brucella Infections in Animals and Man. Commonwealth Foundation, New York.

Jensen, H. L. (1954). The Azotobacteriaceae. Bact. Rev. 18, 195.

Jensen, V. \& Petersen, E. J. (1955). Taxonomic studies on Azotobacter chroococcum Beij. and Azotobacter beijerinckii Lipman. Royal Veterinary and Agricultural College Yearbook, p. 107. Copenhagen.

Pochon, J. \& Barjac, H. (1958). Traité de Microbiologie des Sols. Dunod Ed., Paris.

(Received 5 October 1959) 